\title{
Neonatal Screening for Cystic Fibrosis: A Meta Analysis Study
}

\author{
Ashraf Mahmood ${ }^{1,4,}$, , Divya Rajmohan ${ }^{2,4}$, Shahnawaz Hashmi ${ }^{4}$, Shayan Faiq ${ }^{4}$, \\ Adewale Plumptre ${ }^{3,4}$, Robert Soltes ${ }^{4}$, Zahir Faruqi ${ }^{5}$, Lubna Attal ${ }^{6}$ \\ ${ }^{1}$ Metropolitan Jewish Health System, New York, USA \\ ${ }^{2}$ Weill Cornell Medicine, New York, USA \\ ${ }^{3}$ Department of Public Health, Walden University, Minneapolis, USA \\ ${ }^{4}$ Hameed's Primary Care Clinic, New York, USA \\ ${ }^{5}$ Department of Biochemistry, Long Island University, New York, USA \\ ${ }^{6}$ School of Medicine, St. George University, St. George's, Grenada

\section{Email address:} \\ ashrafpuran@hotmail.com (A. Mahmood),divyans7@gmail.com (D. Rajmohan), shahnawazhashmi76@gmail.com (S. Hashmi), \\ shayan.faiq.93@live.com (S. Faiq),molaayo100@yahoo.com (A. Plumptre), Robert_soltes@yahoo.com (R. Soltes), \\ zfaruqi1999@gmail.com (Z. Faruqi),1fa233@nyu.edu (L. Attal) \\ ${ }^{*}$ Corresponding author
}

\section{To cite this article:}

Ashraf Mahmood, Divya Rajmohan, Shahnawaz Hashmi, Shayan Faiq, Adewale Plumptre, Robert Soltes, Zahir Faruqi, Lubna Attal. Neonatal Screening for Cystic Fibrosis: A Meta Analysis Study. American Journal of Pediatrics. Vol. 5, No. 4, 2019, pp. $200-208$. doi: 10.11648/j.ajp.20190504.16

Received: June 11, 2019; Accepted: September 11, 2019; Published: October 9, 2019

\begin{abstract}
Objective: The main goal of this literature review is to investigate and compare the current screening procedures for the diagnosis of cystic fibrosis in neonates. Cystic Fibrosis or CF is a lethal, genetic disease. The disease is neither too widespread nor too rare. There are more than 30,000 CF patients in the United States, and the number of CF carriers is still unidentified. CF affects more than one organ system in patient's body; due to this, there is no definitive treatment plan for the disease. The cure is dependent on the results of prenatal and postnatal diagnosis. The patients may exhibit all or few symptoms associated with the disease. Sometimes, symptoms are completely absent at the time of birth and become more prevalent with the age. Methods \& Materials: We extensively studied the peer-reviewed scientific journals to understand the existing diagnostic and screening methods for cystic fibrosis. During our research, we kept the focus on newborn screening and evaluated the clinical data from previous studies. We retrieved tables and figures from electronic databases to indicate these results more effectively. We also analyzed the available information on sensitivity, specificity, positive predictive value and negative predictive value of these screening programs. Result \& Conclusion: The data indicates that sensitivity of neonatal screening programs is less than $90 \%$ (or even below $80 \%$ in some cases) when only one test is performed. However, sensitivity increases as the multi-stage approach is adopted. Besides, the CF detection rate is also influenced by multiple factors including ethnicity and age of the patient, duration of the study, type of CFTR mutation, nutritional habits, etc. Many studies need to be carried out to determine optimal cutoff values for both IRT and the sweat test. Genomics and computational biology can be used not only in identifying the other important CFTR mutations, but also in evaluating their impact on the patient's body.
\end{abstract}

Keywords: Cystic Fibrosis, Symptoms, Signs of Cystic Fibrosis, Newborn Screening for Cystic Fibrosis, IRT, CFTR Mutations, Sweat Chloride Tests, Meconium Ileus, Nasal Potential Difference

\section{Introduction}

Cystic fibrosis or Mucoviscidosis is an inherited, life- threatening disease that primarily deteriorates lungs, digestive system, male fertility, and sweat glands. (Mckusick, V.; Cystic Fibrosis) [1-2]. According to statistics, approximately 1 in 2,500 babies born in the United States 
every year are diagnosed with cystic fibrosis $(\mathrm{CF})$; which means, each year 1,700-2,000 babies are born with this condition. [2] Although the disease is more prevalent in American Caucasians or individuals of European descent, the patients with cystic fibrosis (CF) are present all over the world. Nearly 1 in 9,600 Hispanic, 1 in 17,000 African American and 1 in 90,000 Asian Americans infants are diagnosed with $\mathrm{CF}$.

Almost 50 years ago, cystic fibrosis was considered one of the most fatal childhood disorders. Most newborns with CF used to die during first two years of life. The reported median age of the death was four years in 1959. With the advent of new treatments to reduce the gravity of the disease, many patients can now carry their lives without many complications in adulthood. These developments have increased the median age of mortality. The survival data of 1990 showed a remarkable progression in this direction when the average age of death was recorded as 28 years in CF patients.

Before we proceed towards the diagnosis and effective treatment plans for the cystic fibrosis, it is essential to have a profound understanding of the causes and symptoms of the disease. Patients with CF share many symptoms with normal cold and cough and hence, sometimes are not taken seriously. Lack of knowledge about the disease can result in a late diagnosis and sometimes death of newborns.

The individuals with $\mathrm{CF}$ produce abnormally condensed, dehydrated, and sticky mucus that gets accumulated eventually and cause blockage in lungs, pancreas, and other vital organs. Mucus is a lubricated substance that protects the linings of respiratory, gastrointestinal, and reproductive systems. However, overabundance of this substance can obstruct the airways, impair pancreas, and diminish the fertility in males.

$\mathrm{CF}$ is the most common example of an Autosomal recessive disorder. [3] In this type of disorder, an individual inherits two copies of a mutated gene, one from each parent. There is an exorbitant probability that both parents have only one copy of an abnormal gene and have no signs or symptoms of the disease. Such individuals are known as CF carriers; they have no symptoms of the disease, but may transmit the defective genes to their future generations. Research indicates that four out of five individuals with $\mathrm{CF}$ are born in families that have no previous history of the illness. If the child inherits a mutated gene from one parent and a normal gene from the other, then she/he will just be the carrier of CF and show no sign of disease.

$\mathrm{CF}$ is the consequence of a mutation in a single gene termed as Cystic Fibrosis Transmembrane Conductance Regulator (CFTR). This gene is present on chromosome 7 and consists of 230,000 base pairs long sequence. It encodes for a 1,480 amino acid long transmembrane protein, called CFTR protein. [3-4] The protein is spanned in the outer membrane of the cells of lungs, pancreas, the sudoriferous (sweat) glands, and all other exocrine glands. It mediates and regulates the conduction of chloride ion across the plasma membrane and also plays a significant role in the generation of mucus, sweat, and digestive juices. The mutation in the gene produces dysfunctional protein, due to which the production of an operational CFTR protein is inhibited.

There are greater than 1500 known mutations for the gene, and each mutation causes specific disruption of the CFTR protein. Among them, the most common mutation is $\Delta \mathrm{F} 508$, which is responsible for more than half of CF cases. This mutation occurs due to the obliteration of three consecutive T's from the gene sequence that renders loss of an amino acid "phenylalanine" at the 508th position of the protein. A normal CFTR protein ensures free movement of the ions across the membrane, whereas a distorted protein interferes with the normal functioning and restricts these ions from moving freely.

Cystic Fibrosis is an untreatable disorder, but early diagnosis can help in managing the disease and mitigating the severe damages of vital organs and tissues of the body. Today, the mean life expectancy of CF patients is between 42 to 50 years in the developed countries.

To detect the genetic ailments at the earliest, the US government initiated Newborn Screening (NBS) in 1960; a nationwide public health program to screen neonates. [5] Since then, many developments have taken place to improve the efficacy of the program. The major aim of the program is to screen children within 2-3 days after birth to diagnose the possibility of genetic disorders including phenylketonuria, sickle cell anemia, cystic fibrosis, etc. NBS is now imperative in all 50 states as early diagnosis can obviate many of the lethal outcomes. However, the process and methods might vary from state to state.

The widely used neonatal screening methods for cystic fibrosis are IRT (Immunotrypsinogen Test), CFTR mutation analysis, and the sweat chloride test. IRT analyzes the level of trypsinogen enzyme which is usually elevated in CF patients. CFTR analysis is a type of DNA analysis that detects CFTR mutation in both antenatal and postnatal stages. This test has an advantage over IRT as it can identify CF carriers as well.

In 1948, Paul di Sant'Agnese discovered the presence of excessive salt in the sweat of CF patients. ${ }^{7}$ Based on these findings, Gibson \& Cooke developed a test in 1959 to estimate the electrolytes in the patient's sweat. [8] It was an outstanding discovery to diagnose cystic fibrosis. Even today, the test is considered a "Gold Standard Test" for the diagnosis. This test analyzes the chloride level in the newborn's sweat; an increased amount is a sure indicator of the disease. $\mathrm{CF}$ is confirmed when the sweat chloride concentration of two consecutive independent tests is higher than $60 \mathrm{mmol} / \mathrm{L}$. Statistics indicates that almost 98-99\% CF patients have positive sweat test results.

Schutt and Isles (1968) detected an abnormally high amount of albumin in the meconium of CF newborns. [9-10] This laid the foundation of newborn screening for cystic fibrosis. Later on, with further developments in the field, two new methods have been proposed to screen the disease at the early stage. In 1979, Crossley et al. found out the elevated immunoreactive trypsinogen (IRT), a pancreatic enzyme, in the blood of infants diagnosed with $\mathrm{CF}$. [6] The first newborn screening program for CF in New Zealand and New South Wales started in 1981. [7] These screenings also highlighted an abnormally high amount of 
IRT in the blood sample of CF patients. It opened a new pavement for the diagnosis of disease in neonates. Today, by using heel stick method, blood is collected from the heel of an infant on a filter paper called "Guthrie card" (named after Robert Guthrie, who designed this test) and sent to a biochemical laboratory for detailed analysis.

The main advantage of this procedure is that it can be performed soon after the birth and besides IRT level, can also detect most common types of CFTR mutations. If the newborn has an elevated IRT level $(>99.8 \%)$ and possesses two or more CFTR mutations, then the baby is a CF patient. But if the child has elevated IRT but only one CFTR mutation is present in the DNA, then the child is a CF carrier. To confirm the disease, a child is further referred for the sweat test. Over the years, scientists investigated the credibility of these procedures and compared their results to establish the most reliable screening method.

\section{Materials and Methods}

There is a plethora of information available online on cystic fibrosis, but we restricted our research to peer-reviewed journals only to enhance the credibility of the paper. We used electronic databases including OMIM, PubMed, and SciELo. In this study, we excluded the articles related to other genetic diseases, and $\mathrm{CF}$ screening in adults. We didn't include papers that majorly focused on $\mathrm{CF}$ in African or Asian populations because the disease is more prevalent in the Caucasian population. We confined our search to the previous neonatal screening programs and recent developments in this field. The major keywords we included in our investigation are Cystic Fibrosis, newborn screening for cystic fibrosis, IRT test and its efficacy, DNA analysis for cystic fibrosis, the sweat chloride tests, meconium ileus detection, CT scans for cystic fibrosis, and NPD tests, etc. We also referred past literature reviews published in PubMed.

We retrieved thousands of articles based on these keywords and the topic; we selected 40 research papers and 8 review articles for our work. However, after reading the abstract of each paper, we concluded that few of them were not appropriate for our research as they neither focused on newborn screening nor were relevant for current discussions. We didn't include the journals which referenced the historical significance of the disease. Since our primary aim was to analyze and compare the efficiency of each screening method, we chose those journals that had substantial clinical data. Hence, we finally chose 15 research papers and 4 review articles. Based on the data available from past studies, we will analyze and compare the relative efficacy of all these procedures.

We evaluated the results and discussion section of each paper and then summarized the key points in the study. We presented these summaries and correlated them with each other, focusing on the current status, limitations, and future possibilities of each screening method. We also used seven tables and one figure that were directly retrieved from their original source. The tables have been used to compare the sensitivity/specificity, positive predictive value (PPV), and negative predictive value (NPV) of the different screening programs conducted all over the world.

\section{Results}

In 1993, Gregg et al. conducted two screen tests to identify newborns with $\mathrm{CF}$ in Wisconsin. ${ }^{11}$ The first experiment (first tier) included an IRT test (cutoff $>180 \mathrm{ng} / \mathrm{ml}$ ), followed by a sweat test. They screened 220,865 babies for the presence of disease. Almost 369 infants had elevated IRT; 46 out of them were diagnosed as CF patients. Later, 7 more CF patients were detected who had a false negative IRT value. This test had a sensitivity of $87 \%$ and a positive predictive value (PPV) of 12.5\%. [11] The second study (second tier) included IRT/DNA analysis test (IRT level $>100 \mathrm{ng} / \mathrm{ml}$ ), followed by a sweat test. Total 21,258 infants were screened, and 518 out of them had an elevated IRT level; 24 had at least, one copy of mutated $\Delta \mathrm{F} 508$ gene, and 4 had CF. This test had PPV of $16.7 \%$, significantly larger than IRT test if both tests would have included the same number of patients. When they compared the cost of each test, they found out that the cost of IRT/DNA analysis was slightly higher (\$11,374 per CF patient) than the IRT analysis $(\$ 10,187$ per CF patient). As IRT/DNA test had a decrease number of false positive subjects, Gregg concluded that this method was more accurate and cost effective. [11]

There are many proposed protocols for the newborn screening. Some involve one stage screening using meconium or IRT testing with each other or DNA analysis, and others emphasize on multi-stage screening to have an improved positive predictive value. [12-13] The IRT cutoff varies from laboratory to laboratory, depending upon the local population. The second IRT screening is usually not needed if IRT/DNA analysis is used. Besides, if two CFTR mutations are present, then it also precludes the need for the sweat test. Table 1 summarizes the neonatal screening protocols used by the majority of labs.

Table 1. (Murray, J. et al. Screening for Cystic Fibrosis. Health Technology Assessment 1999; Vol. 3, No. 8, page 34-36, Pubmed). Neonatal Screening Protocols.

\begin{tabular}{lll}
\hline Protocol & Stages & Description \\
\hline Meconium & 1 & Meconium 1 Meconium lactase or albumin test only. \\
IRT & 1 & IRT test only. \\
IRT + meconium & 2 & IRT test initially; meconium test if IRT level raised. \\
IRT + IRT & 2 & IRT test initially; the second sample tested for IRT if first is raised. \\
IRT + DNA & 2 & IRT test initially; the first sample tested for CFTR mutations if IRT level is raised. \\
IRT + meconium + IRT & 3 & IRT test initially; meconium test if IRT level is raised and, if positive, refer for sweat testing; IRT test on the second \\
& & sample if meconium test negative, and initial IRT level very high. \\
IRT + DNA + IRT & 3 & IRT test initially; the first sample tested for CFTR mutations if IRT level is raised; IRT test on second sample in \\
\end{tabular}


Neonatal screening programs for cystic fibrosis have been adopted worldwide to reduce overall mortality rate. Tables 2 and 3 summarizes the results of these studies conducted using different sets of population, protocols, and duration.

Table 2. (Murray, J. et al. Screening for Cystic Fibrosis. Health Technology Assessment 1999; Vol. 3, No. 8, page 49-54, Pubmed) Neonatal Screening: Results of 20 programs in the UK and elsewhere.

\begin{tabular}{|c|c|c|c|c|c|}
\hline Study & Protocol & Number & First Positive screened & Sweat test & False-positive \\
\hline \multicolumn{6}{|l|}{ UK } \\
\hline East Anglia & IRT + IRT & 211,344 & 1150 & 99 & 20 \\
\hline Leeds & Mixed & 81,778 & not known & not known & not known \\
\hline Northants & IRT + IRT & 104,000 & 510 & 53 & 23 \\
\hline Northern Ireland & $\mathrm{IRT}+\mathrm{IRT}$ & 108,424 & 5120 & 136 & 102 \\
\hline Trent & IRT + IRT & 311,857 & 1849 & 114 & 26 \\
\hline Wales \& West Midlands & $\mathrm{IRT}+\mathrm{IRT}$ & 227,183 & 944 & 95 & 33 \\
\hline \multicolumn{6}{|l|}{ Elsewhere } \\
\hline Brittany, France & IRT + DNA & 32,300 & 379 & not known & not known \\
\hline Collaborative study, France & IRT + IRT & 513,440 & 5948 & not known & not known \\
\hline Colorado, USA & $\mathrm{IRT}+\mathrm{IRT}$ & 461,364 & 884 & 128 & 74 \\
\hline \multirow[t]{2}{*}{ New South Wales } & IRT + IRT & $1,015,000$ & 7362 & 577 & 335 \\
\hline & IRT + DNA & 189,000 & 1968 & 111 & 102 \\
\hline New Zealand & $\mathrm{IRT}+\mathrm{IRT}$ & 210,751 & 1399 & 101 & 29 \\
\hline Normandy, France & IRT & 79,800 & 253 & 253 & 234 \\
\hline \multirow[t]{2}{*}{ Northeast Italy } & Mixed & 773,206 & not known & not known & not known \\
\hline & IRT + meconium + IRT & 157,992 & 1320 & 215 & 173 \\
\hline Northeast Netherlands & meconium & 94,043 & not known & not known & not known \\
\hline Queensland & IRT + IRT & 180,000 & not known & not known & not known \\
\hline South Australia & $\mathrm{IRT}+\mathrm{DNA}$ & 108,871 & 1220 & 89 & 82 \\
\hline Victoria & IRT + DNA & 130,708 & 1142 & 97 & 82 \\
\hline Vienna, Austria & IRT & 19,992 & 119 & 119 & 108 \\
\hline West Pennsylvania, USA & IRT + IRT & 105,734 & 827 & 201 & 181 \\
\hline \multirow[t]{2}{*}{ Wisconsin, USA } & IRT & 220,865 & 369 & 369 & 323 \\
\hline & IRT + DNA & 104,308 & 2056 & 123 & 113 \\
\hline
\end{tabular}

Table 3. (Murray, J. et al. Screening for Cystic Fibrosis. Health Technology Assessment 1999; Vol. 3, No. 8, page 49-54, Pubmed). Neonatal Screening: Incidence of $C F$ in 20 Programs.

\begin{tabular}{|c|c|c|c|c|}
\hline Study & Meconium ileus & CF: number detected & CF: number missed & CF: detection rate \\
\hline \multicolumn{5}{|l|}{ UK } \\
\hline East Anglia & 18 & 79 & 1 & $99 \%$ \\
\hline Leeds & 5 & 25 & 1 & $96 \%$ \\
\hline Northern Ireland & 12 & 34 & 14 & $71 \%$ \\
\hline \multirow[t]{2}{*}{ Trent } & 23 & 88 & 6 & $94 \%$ \\
\hline & 6 & 44 & 3 & $94 \%$ \\
\hline Brittany, France & not known & 11 & 2 & $85 \%$ \\
\hline Collaborative study, France & 15 & 100 & 7 & $93 \%$ \\
\hline Colorado, USA & 12 & 54 & 7 & $89 \%$ \\
\hline \multirow[t]{2}{*}{ New South Wales } & 80 & 242 & 30 & $89 \%$ \\
\hline & 9 & 53 & 0 & $100 \%$ \\
\hline New Zealand & not known & 72 & 6 & $92 \%$ \\
\hline Normandy, France & 3 & 19 & 1 & $95 \%$ \\
\hline Northeast Italy & 3 & 42 & 0 & $100 \%$ \\
\hline Northeast Netherlands & 4 & 19 & 5 & $79 \%$ \\
\hline Queensland & not known & not known & not known & \\
\hline South Australia & 7 & 33 & 0 & $100 \%$ \\
\hline Victoria & 9 & 18 & 3 & $93 \%$ \\
\hline Vienna, Austria & not known & 11 & 1 & $92 \%$ \\
\hline West Pennsylvania, USA & not known & 20 & 0 & $100 \%$ \\
\hline \multirow[t]{2}{*}{ Wisconsin, USA } & 11 & 46 & 4 & $92 \%$ \\
\hline & 6 & 15 & 0 & $100 \%$ \\
\hline
\end{tabular}


The above tables indicate the variation in the protocols, cutoffs, and duration of the screening. It can be inferred from the tables that the best outcome can be obtained by using a multistage (IRT/DNA or IRT/IRT) screening approach. For example, East Angelia (1982-89) used a two-stage protocol (IRT/IRT). In this study, a total of 1150 patients had been screened positive after the first test and were recommended for the second test; 99 out of them had a positive sweat test. Finally, 79 had been diagnosed with $\mathrm{CF}$, and 20 were reported as false-positives. [14] The other 18 had meconium ileus, and only 1 patient had a falsenegative result. The detection rate of this screening program was $99 \%$. But the data showed great diversity and contradictions in protocols. There were many programs which followed two-stage or mixed protocols but the detection rate was below $90 \%$. Northern Ireland also followed the two stage IRT/IRT protocol, but only $71 \%$ cases had been diagnosed correctly. [12]

This disparity in the detection rate can be caused by many reasons, including the age of the newborn when screened, duration of the study, contamination of the samples, ethnicity of the population, and type of CFTR mutations, etc. It can also be concluded from Table 3 that the results of meconium screening have not been reported in all studies and cannot be used alone for decision making. Meconium ileus may be present due to other disorders as well, and hence, the test can produce more false positive results.

Table 4. (Murray, J. et al. Screening for Cystic Fibrosis. Health Technology Assessment 1999; Vol. 3, No. 8, page 54-56, Pubmed). Neonatal Screening. Summary Performance Indicators according to protocol.

\begin{tabular}{|c|c|c|}
\hline Protocol & Rate & Numbers \\
\hline \multicolumn{3}{|c|}{ First positive rate } \\
\hline One-stage & 2.3 per 1000 & $741: 320,657$ \\
\hline IRT + IRT & 8.0 per 1000 & $25,993: 3,269,097$ \\
\hline IRT + DNA & 12.0 per 1000 & $6765: 565,187$ \\
\hline Three-stage & 7.2 per 1000 & $2046: 283,965$ \\
\hline \multicolumn{3}{|c|}{ False-positive rate } \\
\hline One-stage & 2.1 per 1000 & $665: 320,575$ \\
\hline IRT + DNA & 0.7 per 1000 & $384: 532,745$ \\
\hline Three-stage & 0.6 per 1000 & $175: 283,876$ \\
\hline \multicolumn{3}{|c|}{ Positive predictive value } \\
\hline One-stage & $10 \%$ & $76: 741$ \\
\hline IRT + IRT & $45 \%$ & $681: 1504$ \\
\hline IRT + DNA & $25 \%$ & $139: 559$ \\
\hline Three-stage & $27 \%$ & $86: 322$ \\
\hline One-stage & $90 \%$ & $95: 106$ \\
\hline IRT + IRT & $90 \%$ & $781: 866$ \\
\hline IRT + DNA & $97 \%$ & $150: 155$ \\
\hline Three-stage & $97 \%$ & $86: 89$ \\
\hline
\end{tabular}

Table 4 compares the efficacy of different screening methods and shows that although the CF detection rate is same (97\%) for both IRT/DNA and IRT/DNA/IRT approaches, the two stage (IRT/DNA) method has higher false-positive rate and lower PPV than compared to threestage approach. The table also indicates that when compared to IRT/IRT and IRT/ DNA approach, the first method has lower false positive rate and much higher PPV than the second method. The possible explanations for this can be the presence of either hypertrypsinemia condition in cystic fibrosis carriers or unidentified mutations in the affected newborns. The results are somewhat contradictory and need further study. [12] Usually "Genotype-Phenotype correlations" is used to diagnose the symptoms of the disease; but in the CF case, these correlations are inadequate. The only way to establish this relation is to compare b-IRT level and sweat test results of the newborns having 0-2 mutations in their CFTR gene.

Paracchini et al. (2012) investigated the variation in b-IRT level in infants born in Lombardia. [15] They screened 717,172 infants; out of them, 7,354 were hypertrypsinemic and were divided into four groups for further study:
1) $\mathrm{CF}$ non-carriers.

2) $\mathrm{CF}$ carriers.

3) Patients with CFTR-RD (CFTR-related disorders).

4) Patients with CF.

Although there was a remarkable difference in b-IRT levels among the four groups $(p<0.001)$, the difference between these values for non-carriers and carriers was negligible. The test had high predictive accuracy in differentiating the $\mathrm{CF}$ patients from carriers and non-carriers, but it was not very accurate in determining the differences between four groups (c-index: 0.60). This study showed that there is a link between genotype and phenotype of the disease and further work needs to be done to use this connection in screening.

As described earlier, there are more than 1,500 mutations recorded in CFTR gene. The frequency of these mutations varies according to race and ethnicity. Therefore, before performing the test, it is prudent to know the patient's racial background. There are many mutations which are still unknown or have such a low frequency that until now, no specific method has been developed to screen them.

It is an arduous task to determine which CFTR mutations 
should be considered while newborn screening. Baker and colleagues (2011) compared the Wisconsin CFTR screening results from 1994 to 2008 and determined an optimal mutation panel. [16] They reviewed test data of about 1 million infants and determined that the sensitivity of IRT/DNA method was $96.2 \%$ when only F508del mutation was taken into consideration. However, the sensitivity increased $(97.3 \%)$ when 23 other mutations were included in the screening along with $96^{\text {th }}$ percentile IRT cutoff. To reduce the total number of false negatives, the use of a 23 CFTR mutation panel has been recommended by American College of Medical Genetics (ACMG).

Table 5. (Baker, M. et al. OPTIMAL DNA TIER FOR THE IRT/DNA ALGORITHM DETERMINED BY CFTR MUTATION RESULTS OVER 14 YEARS OF NEWBORN SCREENING. J Cyst Fibros. 2011 Jul; 10 (4): 278-281. PubMed). CF Screening False Negatives and Cause of Delayed Diagnosis after IRT/DNA Analyzes.

\begin{tabular}{lllllll}
\hline Year & Mutation 1 & Mutation 2 & IRT & Age at Diagnosis & Sweat Test Results (mmol/L) & Cause of False Negative \\
\hline 1995 & p. R553X & Unknown & $53(56)$ & 4 months old & 94 & IRT below the cut-off \\
1996 & p. R553X & p. R1161X & $64(56)$ & 6 weeks old & 109 & 108 \\
1997 & p. R347P & Unknown & $82(56)$ & 7 weeks old & 110 & F508del alone as the 2nd tier \\
2000 & $3007 d e l G$ & Unknown & $99(64)$ & 9 months old & 59 & F508del alone as the 2nd tier \\
2001 & Unknown & Unknown & $44(66)$ & 7 years old & 77 & Rare mutations \\
2002 & p. G551D & p. Q1291H & $53(64)$ & 4 years old & IRT below the cut-off \\
2003 & F508del & F508del & $45(51)$ & 1 -year- old & 66 & IRT below the cut-off \\
2004 & p. R170H & Unknown & $13(62)$ & 3 years old & & IRT below the cut-off \\
\hline
\end{tabular}

The table 5 shows that IRT tests produced more false negatives and had low sensitivity. Hence, it is very important to determine an optimal cutoff for IRT as well and use at least CFTR 23 mutations to enhance the sensitivity of the test.

In 2014, Cortés et al. studied the effects of ethnicity, gender, and feeding habits on the IRT value in Spanish children who underwent screening for cystic fibrosis. [17] The Figure 1 shows that IRT value was almost similar for the children who are on breastfeed, formula or mixed supplements whereas there was a drastic increase in the value for the infants who were given special diet.

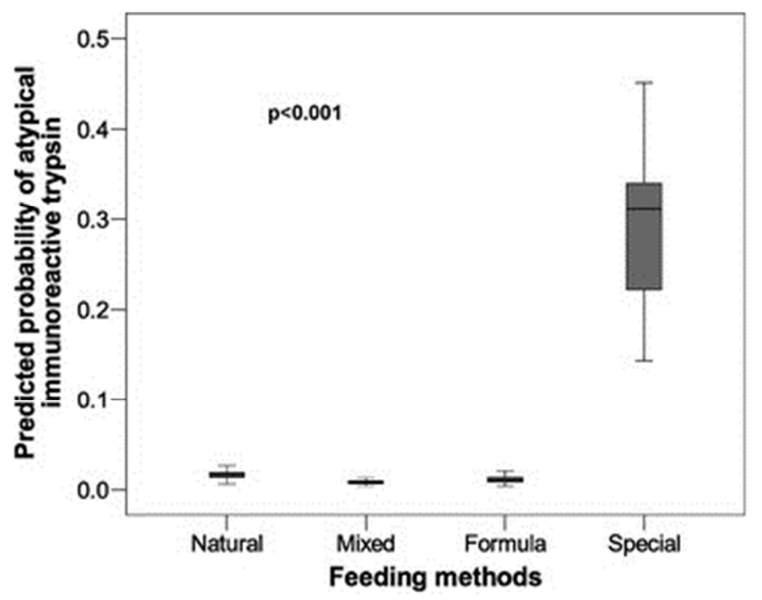

Figure 1. (Cortés, E. et al. Differences in immunoreactive trypsin values between a type of feeding and ethnicity in Neonatal cystic fibrosis screening: a cross-sectional study Orphanet J Rare Dis. 2014; 9: 166. PubMed), Predicted probability of atypical immunoreactive trypsin between feeding methods in newborns (2012-2013 data).
Cortés and colleagues also noticed that IRT value in African newborns was a little higher than their American or Asian counterparts. [18] Besides this, the age of the child and the presence of other pathological conditions were other variables that affected the IRT value drastically. Hence, they concluded that to decrease the number of false positives, it is essential to take these factors into consideration while deciding a cutoff value for the enzyme.

The two most common protocols to diagnose CF are IRT/IRT, followed by a sweat analysis and IRT/ CFTR (or IRT/CFTR/IRT) method, followed by a sweat test. Due to the low sensitivity of IRT test and the presence of many unknown CFTR mutations, the disease can only be confirmed after the sweat test is done, 2-3 weeks after birth.

Mattar AC et al. compared the cost and accuracy of the classic (Gibson \& Cooke) and sweat conductivity test (2010). [19] They found out that the conductivity test had $100 \%$ sensitivity and $100 \%$ specificity whereas the classic test has $95 \%$ of sensitivity and specificity. The conductivity test also costs less than the Gibson \& Cooke method.

Furthermore, Domingos et al. evaluated the sweat test results (performing conductivity method and coulometric measurement) in newborns. [20] The results were outstanding, with $16.7 \%$ false positive rate. All patients had chloride concentration higher than $50 \mathrm{mmol} / \mathrm{L}$. Therefore, the test showed $100 \%$ sensitivity, $96.2 \%$ specificity, $83 \%$ positive predictive value, and $100 \%$ negative predictive value. The quantitative analysis (presented in Table 6 and Table 7) restated that the sweat test is the most decisive test to confirm $\mathrm{CF}$.

Table 6. (Domingos, M. et al. Sweat conductivity and coulometric quantitative test in Neonatal cystic fibrosis screening. Jornal de Pediatria vol. 91 issue 6 , November-December 2015: 590-595. PubMed). Quantitative measurement of chloride and conductivity in patients without CF.

\begin{tabular}{lllll}
\hline & Mean (SD) & 95\% CI & Median & Minimum \\
\hline Chloride $(\mathrm{n}=444)$ & $26.0 \pm 32.2$ & $23.0-29.0$ & 12 & 3 \\
Conductivity $(\mathrm{n}=444)$ & $44.1 \pm 29.5$ & $41.3-46.8$ & 32 & 6 \\
\hline
\end{tabular}

CF, cystic fibrosis; SD, standard deviation; $95 \%$ CI, 95\% confidence interval.

(Chloride and conductivity values are expressed in $\mathrm{mmol} / \mathrm{L}$ ) 
Table 7. (Domingos, M. etal. Sweat conductivity and coulometric quantitative test in Neonatal cystic fibrosis screening. Jornal de Pediatria vol. 91 issue 6 , November-December 2015: 590-595. PubMed) Quantitative measurement of chloride and conductivity in CF patients.

\begin{tabular}{llllll}
\hline & Mean (SD) & 95\% CI & Median & Minimum & Maximum \\
\hline Chloride 1 $(n=90)$ & $91.9 \pm 19.0$ & $87.5-96.3$ & 97 & 60 & 110 \\
Conductivity 1 $(n=90)$ & $102.4 \pm 18.8$ & $98.5-106.3$ & 108 & 53 & 133 \\
Chloride 2 $(n=90)$ & $91.7 \pm 19.0$ & $86.4-96.9$ & 97.5 & 60 & 115 \\
Conductivity 2 $(n=90)$ & $106.4 \pm 18.9$ & $102.6-110.2$ & 110 & 57 & 129 \\
\hline
\end{tabular}

SD, standard deviation; $95 \% \mathrm{CI}, 95 \%$ confidence interval; CF, cystic fibrosis.

(Chloride and conductivity 1 and 2 correspond to the first and second examinations, respectively. Chloride and conductivity values are expressed in $\mathrm{mmol} / \mathrm{L}$ )

Langen estimated the efficiency of Nanoduct sweat test system as compared to Gibson-Cooke method. [21] They suggested that nanoduct is superior, as it has better sensitivity and specificity. The method requires only $3 \mu \mathrm{L}$ of sweat sample and hence, can be performed just after the birth; though further experiments have to be done in this area.

Screening and diagnosis of CF is a challenging task. Sometimes, the result of first sweat test is misleading and more than one sweat test is needed to confirm the disease. Groves and colleagues recently reviewed the 15 years sweat analysis data from "The Children's Hospital" at Westmead. [22] Their studies inferred that it is better to perform repetitive tests for the infants who had intermediate chloride concentration $(30-59 \mathrm{mmol} / \mathrm{L})$ in their sweat along with increased IRT and at least one copy of mutated CFTR gene. The children who showed disease symptoms in later tests are called "delayed CF". The repetitive tests have helped in managing cystic fibrosis and abated the pancreatic insufficiency, airways obstructions, and severity level.

The recent revolution in the medical field has resulted in many new screening techniques that can diagnose the disease at the prenatal stage. There are many methods including CT scans, infant pulmonary function tests, fecal elastase analysis, etc. that can increase the efficiency of any treatment plan.

Prenatal testing is not only helpful for CFTR mutation analysis, but is equivalently useful to detect an echogenic bowel (a condition called meconium ileus or MI) pattern in the developing fetus. [23]

In addition to the above-mentioned procedures, a chest or sinus CT scan is nowadays used for the diagnosis of pulmonary or sinus infections. [7] The chest CT scans can detect inflammation and infection in the lungs and can also locate the blocked airways whereas sinus CT scan is used to diagnose the infection in the sinuses.

Lüth S studied the level of elastase in fecal stool and determined that this test can predict the severity of pancreatic insufficiency and has good sensitivity and specificity. [24] Data suggests that the stool samples of nearly $80 \%$ of the patients have a very negligible concentration of elastases. The ideal range of elastase in the stool is $>200 \mu \mathrm{g}$ elastase $/ \mathrm{g}$ stool, whereas the stool samples of CF patients contain $<100$ $\mu \mathrm{g}$ elastase/g stool. These values are very useful to deduce the severity of pancreatic insufficiency in patients.

Another more recent test to determine the pancreatic insufficiency in neonates is PAP (pancreatic associated proteins) test or PAP/ IRT test (when combined with IRT), conducted by Sarles et al. [23] They found the method useful because it showed promising sensitivity and specificity.

$\mathrm{CF}$ is the result of anomalies in the salt transport system. A dysfunctional CFTR fails to regulate the transport of ions across the plasma membrane. Transport of sodium and chloride ions produces an electric potential across the airways. Knowles MR et al. (1981) calculated the difference in nasal potential by placing the electrodes on the linings of the nose. [25]

NPD testing is very tedious and is not available commercially. This method is mostly used for research purposes. If the test is used for diagnosis, then the results of other screening and diagnostic techniques must be considered along with NPD value before reaching to any conclusions.

\section{Discussion}

Cystic fibrosis is the disease of genes and cannot be prevented completely. The babies who acquire modified genes from parents have this disease since birth. Even then, early diagnosis of the condition is a daunting task. Statistics suggest that only 10 percent of children show symptoms at the time of birth. Moreover, many of the symptoms have resemblance with common childhood ailments. The lack of any specific symptom further procrastinates the treatment. However, with the help of improved screening and diagnostic techniques, the disease can now be detected at a nascent stage. These procedures have mitigated many of the detrimental effects of the disease and increased the total life expectancy of the patients.

There are many factors that affect the efficiency of screening procedures in neonates. No method is sufficient to detect the disease correctly. Because of this, infants have to undergo repetitive screenings. Before conducting the tests, health care providers must obtain written consent from the parents, as it involves taking a blood sample from a newborn. Sometimes, parents have apprehensions and refuse to proceed with the test. Repetitive tests produce an anxiety in parents as well as in babies. The high cost of these tests is another major reason for the negligence. Many families cannot afford even one test and hence, don't come back for repetitive screenings.

As discussed in the previous section, IRT test alone cannot be used to determine the disease as sometimes they fail to differentiate between $\mathrm{CF}$ patients and CF carriers. Besides, IRT can have deviated values due to other pathological conditions as well. The ethnicity, diet, or age of the patient also influences IRT levels. Because of all these reasons, it is 
very difficult to set an ideal cutoff value for the test. Usually, a high cutoff value is set $\left(>96^{\text {th }}\right.$ percentile) to increase the sensitivity and specificity, but this can produce more false negative results. To decrease the error, tests are sometimes repeated many times, which causes inconvenience to both parents and infants.

Some states still do only an IRT test to detect the CF patients, but others have adopted more effective approach by including DNA analysis as part of a screening test. To increase the sensitivity and specificity of the test, it is recommended that a CFTR mutation test must be accompanied by IRT test, followed by a sweat test. American College of Medical Genetics (ACMG) proposed technical standards and guidelines for CFTR mutation testing in 2002 (published in "Genetics in Medicine"). The prime motive of these guidelines is to enhance the quality and validity of laboratory testing. However, these guidelines are theoretical and actual procedure may differ from patient to patient.

The main limitation of CFTR mutation analysis (DNA analysis) is that there are still many more mutations which are unknown and hence, their physiological impact cannot be evaluated. F508 del is the most common mutation present in Caucasians, but different types of mutations can be present in different ethnic populations. Due to this, many infants with CF are falsely identified as disease-free. Though ACMG has proposed a mutation panel for the evaluation of clinical sensitivity and specificity in non-Hispanic population, more studies are needed to detect all other possible mutations. F508 del mutations are primarily associated with pancreatic insufficiency, infertility, or increased chloride level in sweat, but they are unable to indicate serious pulmonary damage. To calculate the pulmonary risk and progression of the disease, other tests must be done along with DNA analysis. CFTR test can be performed during prenatal stage also, but it has some risk for both mother and the fetus.

Clinical sensitivity and specificity of screening tests vary with family history, race, ethnicity, etc. Therefore, it is desirable to obtain patient's complete information before testing. These values are very useful for prenatal and preconceptual screening. Ideally, the test values either confirm or confute the possibility of the disease. For instance, a test value can never be negative for the child who has $\mathrm{CF}$, and can never be positive for a child who doesn't have the disease. It proposes that if the test has $100 \%$ sensitivity, it correctly identifies all the patients with the disease. However, if a test has only $80 \%$ sensitivity, it identifies $80 \%$ patients (true positives) correctly while remaining $20 \%$ of the patients remain unidentified (false negatives). For instance, the research shows that 23 CFTR mutation panel can detect about $88 \%$ of $\mathrm{CF}$ (with one mutation) carriers and approximately $77 \%(88 \% \mathrm{X} 88 \%)$ of CF patients (with two mutations) in non-Hispanic Caucasians.

Even though IRT/CFTR combination increases the overall sensitivity of the test, sweat tests are the landmark to make the final decision. Two most important procedures are Gibson-Cooke method (the classic method) and Conductivity test. In the classic test, an excellent technical understanding of the procedure is needed to collect and analyze the sample accurately. Besides this, the test also requires a biochemical analysis of the sample that makes the process more complex. Because of human inclusion, there are big chances of error. The false-positive or false-negative results may put a question mark on the authenticity of the test. To minimize these errors or accentuate the validity of the results, tests are reiterated many times. Nowadays, most laboratories are using conductivity method over the classic method. This method is more precise, simple, and less time consuming. The chief limitation of both these methods is that they need ample amount of sweat sample to produce accurate results and hence, cannot be performed soon after the birth. Nanoduct sweat testing system can be a solution to this problem, but it needs further research.

The other screening methods including CT scans, ultrasound for meconium ileus, PAP test, fecal elastase test, or NPD test are only useful when combined with the abovementioned procedures. None of the tests can independently diagnose the disease because the symptoms are not unique to $\mathrm{CF}$, and false results can have both physiological and psychological effects on patients. All these screening procedures have improved the quality of life of CF patients. However, more research is needed to establish a welldesigned screening and treatment plan.

\section{Conclusion}

Cystic fibrosis is incurable. However, once the disease is diagnosed, an effective treatment plan can be designed to manage the disease and mitigate the complications. Most of these plans are preventive and can increase the life expectancy. The chief goals are preventing lung and intestinal infections, recommending a nutritious diet plan, removing sticky mucus from lungs, airways, and intestines, impeding dehydration, etc. (Cystic Fibrosis, PubMed).

Airway clearance techniques (ACT) can clear the mucus from lungs and airways. This can be helpful in reducing the lung infections. Many antibiotics can be helpful in treating the bacterial infections. Inhalers can also be utilized to administer the medicine inside the body. CFTR modulator is used to fix the CFTR protein. It helps in normal ion transport and decreases the other complexities as well. Lung transplantation is also common in the case of serious lung damages. Along with these treatments, regular exercise and physical activities play a significant role in the improvement of the disease. The patients who exercise daily have better lung functioning and stronger bones. These patients are less vulnerable to diabetes or heart diseases

Presentation of a vast number of known and unknown mutations in CFTR gene is the biggest challenge in designing the improved screening and diagnostic techniques as well as in developing potent medicines. All over the world, scientists are working arduously to find the solutions. The commencement of genomics, proteomics, and other molecular biology techniques has shown many positive results in this area. Already, there is a sharp decline in the 
mortality rate caused due to $\mathrm{CF}$ and the future looks much more promising.

\section{References}

[1] Mckusick, V., Cystic Fibrosis. OMIM (\# 219700).

[2] Cystic Fibrosis. Genetic Home Reference. Aug. 2012.

[3] Cystic Fibrosis. PubMed. June 2014.

[4] Bobadilla JL. Cystic fibrosis: a worldwide analysis of CFTR mutations--correlation with incidence data and application to screening. Hum Mutat. 2002 Jun; 19 (6): 575-606. PubMed.

[5] Newborn Screening. Centers for Disease Control and Prevention.

[6] Crossley JR, Elliott RB, Smith PA. Dried-blood spot screening for cystic fibrosis in the newborn. Lancet. 1979; 1: 472-4. PubMed.

[7] Mishra, A. et al. The Relevance of Sweat Testing for the Diagnosis of Cystic Fibrosis in the Genomic Era. Clin Biochem Revv. 26 (4); 2005 Nov PMC1320177. PubMed.

[8] Gibson, L. \& Cooke, R. A TEST FOR CONCENTRATION OF ELECTROLYTES IN SWEAT IN CYSTIC FIBROSIS OF THE PANCREAS UTILIZING PILOCARPINE BY IONTOPHORESIS. PediatricsMarch 1959, VOLUME 23/ISSUE 3. PubMed.

[9] Wildhagen, M., Kate, L., \& Habbema, J. Br Med Bull (1998) 54 (4): 857-875. Screening for cystic fibrosis and its evaluation. Pubmed.

[10] Schutt, W. H. Isles TE. Arch Dis Child 1968; 43: 178-81. Protein in meconium from meconium ileus.

[11] Gregg, R. et al. Application of DNA Analysis in a Populationscreening Program for Neonatal Diagnosis of Cystic Fibrosis (CF): Comparison of Screening Protocols. Am. J. Hum. Genet. 52: 616-626, 1993. PubMed.

[12] Murray, J. et al. Screening for Cystic Fibrosis. Health Technology Assessment 1999; Vol. 3, No. 8, page 35-56, Pubmed.

[13] Heeley, A. F., Bangert, S. K. Ann ClinBiochem 1992; 29: 361-76. The neonatal detection of cystic fibrosis by measurement of immunoreactive trypsin in blood.
[14] Lewis, FA, Cross P., Sehmi, I., Cuckle, H., Quirke, P. J Pathol 1993; 170: A34. Population screening for the cystic fibrosis gene using fluorescent PCR [abstract].

[15] Paracchini, V. et al. Cystic Fibrosis Newborn Screening: Distribution of Blood Immunoreactive Trypsinogen Concentrations in Hypertrypsinemic Neonates. JIMD Rep. 2012; 4: 17-23. PubMed.

[16] Baker, M. et al. OPTIMAL DNA TIER FOR THE IRT/DNA ALGORITHM DETERMINED BY CFTR MUTATION RESULTS OVER 14 YEARS OF NEWBORN SCREENING. J Cyst Fibros. 2011 Jul; 10 (4): 278-281. PubMed.

[17] Cortés, E. et al. Differences in immunoreactive trypsin values between the type of feeding and ethnicity in Neonatal cystic fibrosis screening: a cross-sectional study Orphanet $J$ Rare Dis. 2014; 9: 166. PubMed.

[18] Lewis PA. et al. The Epidemiology of Cystic Fibrosis. In: Hodson ME, Geddes DM, editors. Cystic Fibrosis. 2. London: Arnold; 2000. pp. 13-25. PubMed.

[19] Matter AC et al. Comparison between classic Gibson and Cooke technique and sweat conductivity test in patients with and without cystic fibrosis. J Pediatr (Rio J). 2010 Mar-Apr; 86 (2): 109-14. SciELO.

[20] Domingos, M. et al. Sweat conductivity and coulometric quantitative test in Neonatal cystic fibrosis screening. Jornal de Pediatria vol. 91 issue 6, November-December 2015: 590595. PubMed.

[21] Vernooij, A. et al. Clinical evaluation of the Nanoduct sweat test system in the diagnosis of cystic fibrosis after newborn screening. European Journal of Pediatrics August 2015, Volume 174, Issue 8, pp 1025-1034. PubMed.

[22] Groves, T. et al. Long-Term Outcomes of Children with Intermediate Sweat Chloride Values in Infancy. $J$ Pediatr. 2015 Jun; 166 (6): 1469-74. e1-3. PubMed.

[23] Rodrigues, R. et al. Cystic fibrosis and neonatal screening. Cad. SaúdePública vol. 24 suppl. 4 Rio de Janeiro 2008. PubMed.

[24] Scand, L. et al. Fecal elastase-1 determination: 'gold standard' of indirect pancreatic function tests? J Gastroenterol 2001 Oct; 36 (10): 1092-9. PubMed.

[25] Knowles MR. et al. Measurements of nasal transepithelial electric potential differences in normal human subjects in vivo. Am Rev Respir Dis. 1981 Oct; 124 (4): 484-90. PubMed. 